\title{
Research on the influence of dimension and location of reflective film on the resonance frequency of quartz tuning fork
}

\author{
Zhouqiang Zhang ${ }^{1}$, Shuhai $\mathrm{Jia}^{1, \mathrm{a}}$, Binshan $\mathrm{Ma}^{1}$, Hualing Chen ${ }^{1}$ \\ School of mechanical engineering, Xi'an Jiaotong University Xi'an, Shannxi, China
}

\begin{abstract}
We studied the effected of dimension and location of reflective film on the resonance frequency. Simulation results indicate that the location of reflective film has a greater impact on the resonance frequency of QTF. The higher the position of reflective film, the lower the resonance frequency of QTF. Furthermore, the resonance frequency can also be affected by the dimension of reflective film. However, the reflective film in the middle of the QTF arm is not sensitive to the dimension of reflective film. The frequency is close to the resonance frequency of the QTF model without reflective film, it is about $30259 \mathrm{~Hz}$. We can increase the length and width of reflective film to improve the laser reflection on the QTF surface. Therefore, this position is suitable for the detection of photo-acoustic spectroscopy. The analysis results provide a theoretical basis for researching new photo-acoustic spectrum remote sensing device.

Keywords: Resonance frequency; Location of reflective film; Dimension of reflective film; Quartz tuning fork
\end{abstract}

\section{Introduction}

Photo-acoustic spectroscopy (PAS) is a unique spectral measurement method [1] that utilizes light absorption properties of the test object. The non-radiative deexcitation of energy absorbed by a sample is transformed into the heat energy due to the optical transitions. Through detecting sound waves created by the heat energy, we can obtain the substance spectral information.

At present, microphone, bi-material cantilever and quartz tuning fork (QTF) are usually used as Photoacoustic detectors. Early in the 1970s and 1980s, microphone has been used to detect the photo-acoustic signal of sample [2-4], however, it is easily interfered by air disturbances that will lead to a low sensitivity. In order to overcome the effects of air turbulence, Silicon nitride or silicon cantilevers evaporated chromium and gold with different thicknesses are used to obtain the photo-thermal deflection spectroscopy by Adam R. Krause[5], C. W. Van Neste[6] et al. Compared with microphone, it can improve the detection sensitivity of photo-acoustic signal, and reduce the influence of air disturbances, but is easily affected by ambient temperature. Quartz tuning fork (QTF) is a new photo-acoustic detector with superior performance. The high quality factor and resonance frequency can overcome the effect caused by air turbulence and ambient temperature. It not only improves the detection sensitivity to a large extent by utilizing the piezoelectric effect of QTF, but also realizes remote detection [7-9]. The disadvantage of this method is that the pre-amplifier circuit must be closer to QTF, otherwise, the piezoelectric signal will be drowned by noise because of the long distance. Furthermore, the external electromagnetic filed can interfere with the pre-amplifier circuit, and make signal-noise-ratio (SNR) reduced [10]. The SNR of detection system is proportional to the square root of the resonance quality factor $\left(Q^{1 / 2}\right)$, and the quality factor and resonance frequency of QTF are respectively $10^{4} \sim 10^{5}$ and $32.768 \mathrm{kHz}$, the external air turbulence is difficult to excite the QTF resonance. Therefore, it is a good way to detect the photo-acoustic signal by utilizing the resonance characteristic of QTF. It not only effectively improves the SNR of detection, but also avoid the influence of external electromagnetic interference.

According to the research of literature [11], the resonance amplitude of QTF is about pm. Because of the simple structure, fast response and high sensitivity etc., the laser triangulation can be used to realize microamplitude detection. A new photo-acoustic spectrum remote sensing device is shown in figure 1. The optical path is extended to realize micro-amplitude detection under pm magnitude. In fact, the bad reflective properties of the QTF surfaces and larger laser spot aren't conducive to reflect laser. A better way to improve the reflective properties is to paste reflective film on the surface of QTF arm. The reflective film structure size and film location are the main factors influencing the QTF resonance frequency and SNR. Therefore, it is highly significant in research of the reflective film structure size and film location for improving the SNR of this detection system.

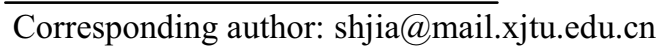




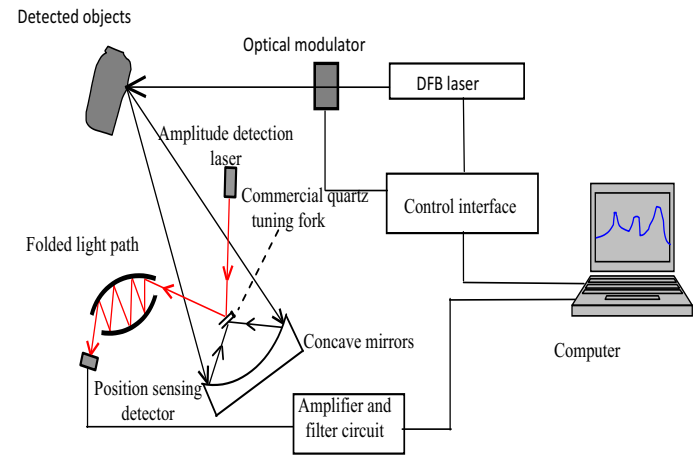

Fig.1 The principle diagram of new photo-acoustic spectrum remote sensing device

\section{Relationship between resonance frequency of QTF and reflective film structure size}

The QTF is a vital part of the photo-acoustic spectrum remote sensing device. Because it is difficult to make a symmetric vibration with sound waves in the surrounding environment at high resonance frequency. Thus the effects of environmental and other frequency noise can be reduced for a narrow spectrum and a high qualityfactor.Therefore, it is suitable to be used as a detect ion sensor. The QTF dimensions are shown in Figure 2, where $L$ is the total length of the tuning fork, $L_{1}$ is the length of the fork arms, $h$ is the thickness of the arms, $b$ is the width of the arms, and $t$ is the gap between the arms. When the reflective film is pasted on the one arm surface of QTF, the direction of reflective film length is consistent with the thickness $h$ of the arms.

The QTF model dimensions in the simulation analysis are as follow:

$$
\begin{aligned}
& L=6 \mathrm{~mm}, L_{1}=4, t=0.22 \mathrm{~mm}, b=0.64 \mathrm{~mm}, \\
& h=0.25 \mathrm{~mm}
\end{aligned}
$$

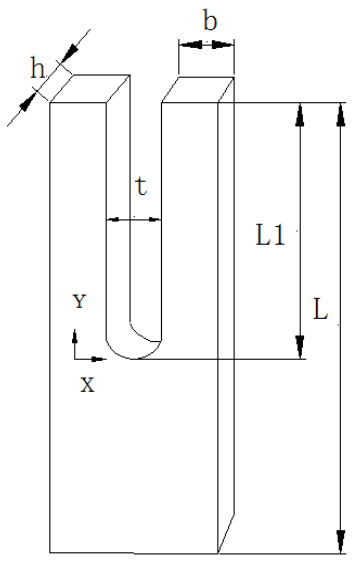

Fig.2 Tuning fork structure diagram

Through the analysis on the resonance frequency of the QTF model without reflective film, it is about $30259 \mathrm{~Hz}$

For optically excited QTF, the natural resonance frequency and vibration mode are major factors. They are primarily dependent on the dimensions and location of the reflective film. Symmetric vibrations must be produced to create an effective piezoelectric response. The symmetric vibrations modes of QTF is shown in Figure3. Therefore, it is critical to examine the relationship between the dimensions and location of the reflective film and the resonance frequency of symmetric vibrations mode. COMSOL software is used to investigate the effect of geometric parameters and location of reflective film on the resonance frequency of symmetric vibrations mode.

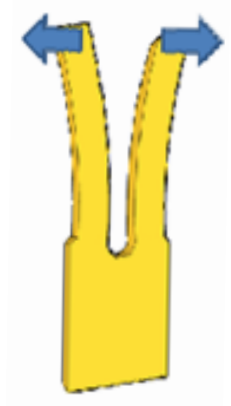

Fig.3 Illustration of symmetric vibrations modes of a tuning fork

\subsection{Changging the length and location of reflective film}

In this paper, resonance frequency change laws of symmetric vibrations mode are studied by changing the length and location of reflective film. According to the Figure 4 , we can found that, when the width and thickness of reflective film is $1 \mathrm{~mm}$ and $0.01 \mathrm{~mm}$ respectively, changing the length of reflective film in the top of the QTF will make the resonance frequency of symmetric vibrations mode lower than QTF without reflective film, and the frequency valley will be generated at the length of $1.5 \mathrm{~mm}$. When the reflective film is in the middle or at the bottom of the QTF arm, the resonance frequency of symmetric vibration will be increased, and the frequency valley at the length of $1.5 \mathrm{~mm}$ will also be reduced.

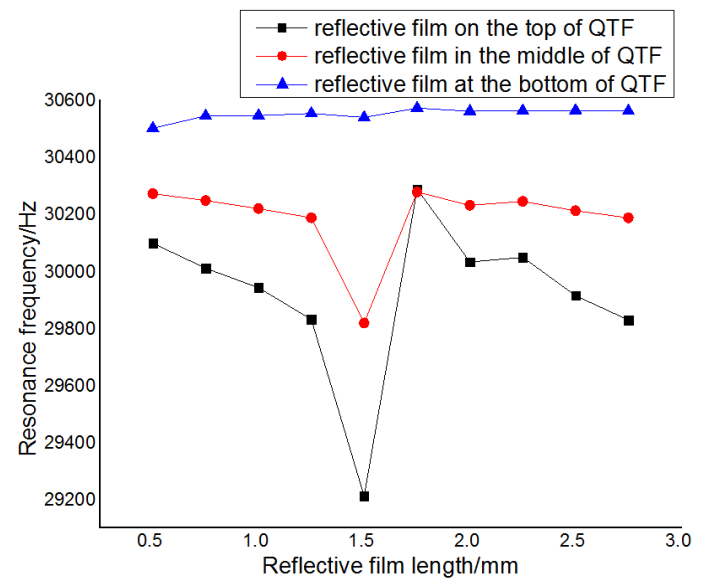

Fig. 4 resonance frequency of symmetric vibrations mode varies with the length of reflective film, when the width of reflective film is $1 \mathrm{~mm}$ 


\subsection{Changing the width of reflective film}

As for the reflective film with the length of $0.5 \mathrm{~mm}$ or $1 \mathrm{~mm}$ and the thickness of $0.01 \mathrm{~mm}$, the same method is used to change the width of reflective film. We can get the curve that the resonance frequency is changing with the width of reflective film. According to the Figure 5 and Figure 6, comparing with QTF without reflective film, we can found that the resonance frequency of symmetric vibrations mode is significantly reduced when the film is located at the top of QTF arm, and the frequency valley will be generated at the width of $1.75 \mathrm{~mm}$. The longer length of reflective film, the greater frequency valley. When the reflective film is located in the middle or at the bottom of the QTF arm, the resonance frequency of QFT will be increased, and the frequency valley will not be appeared.

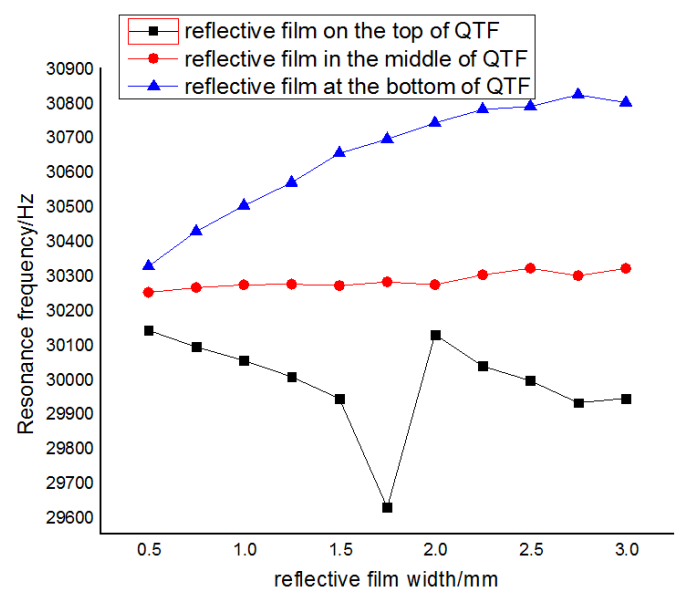

Fig.5 resonance frequency of symmetric vibrations mode varies with the width of reflective film, when the length of reflective film is $0.5 \mathrm{~mm}$

\section{错误！未找到引用源。}

Fig.6 resonance frequency of symmetric vibrations mode varies with the width of reflective film, when the length of reflective film is $1 \mathrm{~mm}$

\subsection{Changing the thickness of reflective film}

Considering the effect of the thickness of reflective film, the same method is used to change the thickness of reflective film with the length and width of $1 \mathrm{~mm}$. We can get the curve that the resonance frequency is changing with the thickness of reflective film. As shown in Figure7, When the reflective film is located in the middle and at the top of the QTF arm, the resonance frequency of QFT will reduce about $200 \mathrm{~Hz}$ and $700 \mathrm{~Hz}$ respectively. However, the reflective film at the bottom of the QTF arm will be advantageous in improving the resonance frequency of QTF, the frequency will increase about $1400 \mathrm{~Hz}$.

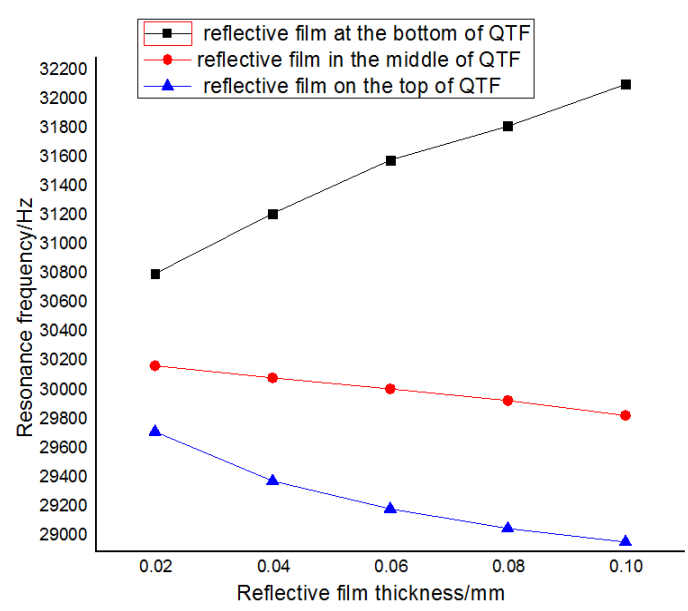

Fig. 7 resonance frequency of symmetric vibrations mode varies with the thickness of reflective film, when the length and width of reflective film is $1 \mathrm{~mm}$

\section{Conclusions}

We studied the effected of size and location of reflective film on the resonance frequency. Simulation results indicate that the location of reflective film has a greater impact on the resonance frequency of QTF .The higher the position of reflective film, the lower the resonance frequency of QTF. Furthermore, the resonance frequency can also be affected by the dimension of reflective film. However, the reflective film in the middle of the QTF arm is not sensitive to the dimension of reflective film. The frequency is close to the resonance frequency of the QTF model without reflective film, it is about $30259 \mathrm{~Hz}$. We can increase the length and width of reflective film to improve the laser reflection on the QTF surface. Therefore, this position is suitable for the detection of photo-acoustic spectroscopy. The analysis results provide a theoretical basis for researching new photo-acoustic spectrum remote sensing device

\section{Acknowledgments}

This work was supported by the National Natural Science Fund Committee and the Civil Aviation Administration of China Jointly Funded Project (U1233116) and Research Fund for the Doctoral Program of Higher Education of China (20120201110032)

\section{References}

1. Veronika Hanyecz;Árpád Mohácsi;Sándor Puskás; Árpád Vágó and Gábor Szabó. Photoacoustic spectroscopy-based detector for measuring benzene and toluene concentration in gas and liquid samples. Measurement Science and Technology 22(12) (2011) : 125602

2. Allan Rosencwaig. Photoacoustic spectroscopy of solids[J]. Physics Today. 28(9)1975:23-30.

3. Allan Rosencwaig. Photoacoustic spectroscopy of solids[J]. The Journal of the Acoustical Society of America.58(suppl 1) 1975: S52. 
4. YUAN Chang-ying,LIU Xian-yong,MENG Gui,etal. Theoretical Study on Radial Resonance Coupling of Cylindrical Photoacoustic Cells [J]. Spectroscopy and Spectral Analysis. 2010, 30(4) : 879-882.

5. Adam R. Krause, Charles Van Neste, Larry Senesac, Thomas Thundat, and Eric Finot.Trace explosive detection using photothermal deflection spectroscopy[J]. JOURNAL OF APPLIED PHYSICS. 2008, 103 (9): 94906-94911

6. C. W. Van Neste; L. R. Senesac; D. Yi and T. Thundat. Standoff detection of explosive residues using photothermal microcantilevers [J]. Applied Physics Letters. 2008, 92 (13): 134102-134104.

7. A.A.Kosterev, F.K.Tittel.Ammonia detection by use of quartz-enhanced photoacoustic spectroscopy with a near-IR tetecommunication diode laser [J]. Appl. Opt. 2004,43:6213-6217.

8. C. W. Van Neste; L. R. Senesac and T. Thundat. Standoff photoacoustic spectroscopy [J].Applied Physics Letters.2008, 92 (23): 234102-234104.

9. C. W. Van Neste;L. R. Senesac and T. Thundat .Standoff Spectroscopy of Surface Adsorbed Chemicals[J]. Analytical Chemistry. 2009,81(5): 1952-1956.

10. LiuYan-Yan,DongLei,WuHong-peng,ZhengHua-Dan, MaWei-Guang,ZhangLei,YiWang-Bao,JiaSuo-

Tang.All optical quartz-enhanced photoacoustic spectroscopy[J]. Acta Phys.sin. 2013, 62(22): 220701

11. Ma Bin shan, Jia Shu hai, Zhang Zhou qiang. Vibration Analysis and Measurement for quartz tuning fork under laser irradiation [J]. Journal of Xi'an Jiao tong University. 2014,48 (10):49-53. 